\title{
Dual-affinity B7-H3/CD3-targeted Protein MGD009
}

National Cancer Institute

\section{Source}

National Cancer Institute. Dual-affinity B7-H3/CD3-targeted Protein MGD009. NCI

Thesaurus. Code C136983.

An Fc-bearing humanized bispecific dual-affinity re-targeting (DART) protein composed of Fv regions derived from monoclonal antibodies against the immunoregulatory protein B7-homologue 3 (B7-H3, CD276) and the T-cell surface antigen CD3, with potential immunostimulating and antineoplastic activities. Upon administration of the MGD009 DART protein, the anti-B7-H3 component targets and binds to the cell surface antigen B7-H3; at the same time, the anti-CD3 component binds to human CD3. This cross-links the T-cells to B7-H3-expressing tumor cells, activates and redirects endogenous T-cells to kill B7-H3-expressing tumor cells, and inhibits proliferation of B7-H3-expressing tumor cells. B7-H3, a type I transmembrane protein and a member of the B7 co-stimulatory protein superfamily, is overexpressed on certain tumor cell types and on various immune cells but is minimally expressed by normal human tissues. B7-H3 is a negative regulator of T-cell activation and its overexpression plays a key role in immuno-evasion, tumor cell invasion and metastasis, and its expression is correlated with poor prognosis. 